\title{
RESIDUALLY FINITE DIMENSIONAL C*-ALGEBRAS AND SUBQUOTIENTS OF THE CAR ALGEBRA
}

\author{
MARIUS DADARlat
}

\begin{abstract}
It is proved that the cone of a separable nuclearly embeddable residually finite-dimensional $\mathrm{C}^{*}$-algebra embeds in the CAR algebra (the UHF algebra of type $2^{\infty}$ ). As a corollary we obtain a short new proof of Kirchberg's theorem asserting that a separable unital $\mathrm{C}^{*}$-algebra $A$ is nuclearly embeddable if and only there is a semisplit extension $0 \rightarrow J \rightarrow E \rightarrow A \rightarrow 0$ with $E$ a unital $\mathrm{C}^{*}$-subalgebra of the CAR algebra and the ideal $J$ an AF-algebra. The new proof does not rely on the lifting theorem of Effros and Haagerup.
\end{abstract}

\section{Introduction}

Throughout the paper we let $B$ denote the CAR algebra, $B \cong \bigotimes_{i=1}^{\infty} M_{2}(\mathbb{C})$. A $C^{*}$-algebra $A$ is called nuclearly embeddable if there is a nuclear faithful representation $\sigma: A \rightarrow \mathcal{L}(\mathcal{H}),[\mathrm{Vo} 2]$. S. Wassermann [W2] has shown that any nuclearly embeddable $\mathrm{C}^{*}$-algebra is exact. By a remarkable theorem of Kirchberg, the converse is also true: any exact $\mathrm{C}^{*}$-algebra is nuclearly embeddable [Ki2]. Having reduced the study of exact $\mathrm{C}^{*}$-algebras to that of nuclearly embeddable $\mathrm{C}^{*}$-algebras, Kirchberg proves the following.

Theorem 1.1. (Kirchberg [Ki2]) Let $A$ be a separable unital $C^{*}$-algebra. The following conditions are equivalent: (i) $A$ is nuclearly embeddable. (ii) There is a semisplit essential extension of $C^{*}$-algebras $0 \rightarrow J \rightarrow E \rightarrow A \rightarrow 0$ such that $E$ is a unital $C^{*}$-subalgebra of the $C A R$ algebra $B$ and $J$ is an AF-algebra stably isomorphic to $B$. (iii) There is an extension of $C^{*}$-algebras $0 \rightarrow J \rightarrow E \rightarrow A \rightarrow$ 0 such that $E$ is a unital $C^{*}$-subalgebra of the $C A R$ algebra $B$.

The equivalence between (i) and (iii) proves that nuclear embeddability (hence exactness) passes to quotients. On the other hand, using the equivalence between (i) and (ii), and his Weyl-von Neumann-Voiculescu type theorem, Kirchberg proved that any separable unital nuclearly embeddable $\mathrm{C}^{*}$-algebra embeds as a unital $\mathrm{C}^{*}$-subalgebra of the Cuntz algebra $\mathcal{O}_{2}[\mathrm{Ki} 4]$. See also $[\mathrm{KPh}]$ for a different proof. Using ideas from [Ki1, Ki2], S. Wassermann [W2] gave a proof of Theorem 1.1 which is shorter and somewhat simpler than the original proof of [Ki2] as it avoids the use of Kirchberg's theory of normalizers of operator subsystems of $C^{*}$-algebras. Both proofs use techniques of operator spaces and

Received March 21, 2000.

The author was supported in part by NSF Grant \#DMS-9970223. 
they rely on the lifting results of Effros and Haagerup [EK] for establishing the equivalence of (i) and (ii).

A separable $\mathrm{C}^{*}$-algebra $A$ is called residually finite-dimensional (abbreviated $R F D$ ) if it has a separating sequence of finite dimensional representations. Equivalently $A$ embeds in a $\mathrm{C}^{*}$-algebra of the form $\prod_{n=1}^{\infty} M_{k(n)}$, where $M_{k}$ stands for $M_{k}(\mathbb{C})$. In this paper we prove that the cone $C F=C_{0}(0,1] \otimes F$ of any separable residually finite-dimensional nuclearly embeddable $\mathrm{C}^{*}$-algebra $F$ embeds in the CAR algebra (Theorem 2.6). Let $A$ be a separable unital nuclearly embeddable $\mathrm{C}^{*}$-algebra. Using the quasidiagonality of $C A$ proved by Voiculescu [Vo3], we observe that there is a semisplit extension $0 \rightarrow I \rightarrow \widetilde{C F} \rightarrow A \rightarrow 0$ with $F$ a separable RFD nuclearly embeddable $C^{*}$-algebra (Lemma 3.1). Combining Theorem 2.6 and Lemma 3.1, we obtain a short proof of the equivalence of (i) and (ii). The implication (iii) $\Rightarrow$ (ii) follows from [EK, Proposition 5.3 and Theorem 3.4], while (ii) $\Rightarrow$ (iii) is obvious. Since a subalgebra of a nuclear $\mathrm{C}^{*}$-algebra is nuclearly embeddable, the non nuclearly embeddable RFD algebras (such as the full $\mathrm{C}^{*}$-algebra of the free group on two generators) are not AF-embeddable. Thus one cannot infer AF-embeddability from just the mere abundance of finite dimensional representations. In a forthcoming paper [D4], we give more general results on the UHF-embeddability of a nuclearly embeddable RFD algebra. Thus we prove that if $A$ is a separable nuclearly embeddable RFD algebra such that either the rational K-homology group $K^{0}(A) \otimes \mathbb{Q}=K K(A, \mathbb{C}) \otimes \mathbb{Q}$ is finitely generated (as a $\mathbb{Q}$-module) or $A$ satisfies the universal coefficient theorem of $[\mathrm{RS}]$ (UCT) for the Kasparov groups, then $A$ embeds in a UHF algebra. Since the proofs of those results rely on certain techniques of KK-theory [Ka, Sk, DE], we have chosen to present here a self-contained elementary proof of the UHFembeddability of $C A$. Previous results on the AF-embeddability of nuclear RFD $\mathrm{C}^{*}$-algebras have appeared in [D2], for $A$ homotopically dominated by an $\mathrm{AF}$ algebra, and [L], for $A$ satisfying the UCT.

\section{Embedding RFD algebras in the CAR algebra}

Proposition 2.1. Let $A, B$ be unital $C^{*}$-algebras and let $\varphi_{0}, \varphi_{1}: A \rightarrow B$ be two unital $*$-homomorphisms which are homotopic. Then for any $\mathcal{F} \subset A$ a finite subset and any $\epsilon>0$ there exist $n \in \mathbb{N}$, a unital $*$-homomorphism $\eta: A \rightarrow$ $M_{n-1}(B)$ and a unitary $u \in U_{n}(B)$ such that

$$
\left\|u\left(\varphi_{0}(a) \oplus \eta(a)\right) u^{*}-\varphi_{1}(a) \oplus \eta(a)\right\|<\epsilon, \quad a \in \mathcal{F} .
$$

Proof. By assumption there is a family of unital $*$-homomorphisms $\left(\varphi_{t}\right): A \rightarrow B$ such that $\varphi_{0}, \varphi_{1}$ are equal to the given ones and for each $a \in A$, the map $t \mapsto \varphi_{t}(a)$ is norm-continuous on $[0,1]$. By uniform continuity we find an integer $n$ such that

$$
\left\|\varphi_{i+1 / n}(a)-\varphi_{i / n}(a)\right\|<\epsilon, \quad 0 \leq i \leq n-1, a \in \mathcal{F}
$$


Define $\eta=\varphi_{1 / n} \oplus \varphi_{2 / n} \oplus \cdots \oplus \varphi_{n-1 / n}$. Using (2)

$$
\left\|\varphi_{0}(a) \oplus \eta(a)-\eta(a) \oplus \varphi_{1}(a)\right\| \leq \sup _{0 \leq i \leq n-1}\left\|\varphi_{i+1 / n}(a)-\varphi_{i / n}(a)\right\|<\epsilon, \quad a \in \mathcal{F} .
$$

If $u \in M_{n}\left(\mathbb{C} 1_{B}\right)$ is the cyclic shift of order $n$, then $u\left(\eta(a) \oplus \varphi_{1}(a)\right) u^{*}=\varphi_{1}(a) \oplus$ $\eta(a)$. From this and (3) we obtain (1).

The following proposition is an easy consequence of [DE, Theorem 3.8]. We give here an alternative proof which does not use KK-theory. Let $\mathcal{L}(\mathcal{H})$ denote the linear operators acting on a Hilbert space $\mathcal{H}$ and let $\mathcal{K}(\mathcal{H})$ denote the compact operators. We have $\mathcal{L}\left(\mathbb{C}^{k}\right)=\mathcal{K}\left(\mathbb{C}^{k}\right) \cong M_{k}$.

Proposition 2.2. Let $A$ be a unital separable $C^{*}$-algebra and let $\varphi, \psi: A \rightarrow M_{m}$ be two unital $*$-homomorphisms which are homotopic. Let $\mathcal{F} \subset A$ be a finite subset and let $\epsilon>0$. Then for any faithful unital representation $\sigma: A \rightarrow \mathcal{L}(\mathcal{H})$ with $\sigma(A) \cap \mathcal{K}(\mathcal{H})=\{0\}$, there is a unitary $v \in \mathbb{C}_{\mathbb{C}^{m} \oplus \mathcal{H}}+\mathcal{K}\left(\mathbb{C}^{m} \oplus \mathcal{H}\right)$ such that

$$
\left.\| v(\varphi(a) \oplus \sigma(a)) v^{*}-\psi(a) \oplus \sigma(a)\right) \|<\epsilon, \quad a \in \mathcal{F} .
$$

Proof. By Proposition 2.1, there is a unital finite-dimensional representation $\eta: A \rightarrow M_{r}$ and a unitary $u \in M_{m+r}$ satisfying

$$
\left\|u(\varphi(a) \oplus \eta(a)) u^{*}-\psi(a) \oplus \eta(a)\right\|<\epsilon / 3, \quad a \in \mathcal{F} .
$$

Let $z$ be the unitary $z=u \oplus 1_{\mathcal{H}} \in \mathbb{C} 1_{\mathbb{C}^{m+r} \oplus \mathcal{H}}+\mathcal{K}\left(\mathbb{C}^{m+r} \oplus \mathcal{H}\right)$. It follows from (5) that

$$
\left\|z(\varphi(a) \oplus \eta(a) \oplus \sigma(a)) z^{*}-\psi(a) \oplus \eta(a) \oplus \sigma(a)\right\|<\epsilon / 3, \quad a \in \mathcal{F} .
$$

By Voiculescu's Theorem [Vo1] there is a unitary $w: \mathcal{H} \rightarrow \mathbb{C}^{r} \oplus \mathcal{H}$ such that

$$
\left\|w \sigma(a) w^{*}-\eta(a) \oplus \sigma(a)\right\|<\epsilon / 3, \quad a \in \mathcal{F} .
$$

If we set $v=\left(1_{\mathbb{C}^{m}} \oplus w^{*}\right) z\left(1_{\mathbb{C}^{m}} \oplus w\right) \in \mathbb{C}_{\mathbb{C}^{m} \oplus \mathcal{H}}+\mathcal{K}\left(\mathbb{C}^{m} \oplus \mathcal{H}\right)$, then (4) follows from (6) and (7). Indeed

$$
\begin{aligned}
\left\|v(\varphi(a) \oplus \sigma(a)) v^{*}-\psi(a) \oplus \sigma(a)\right\| & =\| z\left(\varphi(a) \oplus w \sigma(a) w^{*}\right) z^{*} \\
& \left.-\psi(a) \oplus w \sigma(a) w^{*}\right) \| \\
& \leq 2\left\|w \sigma(a) w^{*}-\eta(a) \oplus \sigma(a)\right\| \\
& +\| z(\varphi(a) \oplus \eta(a) \oplus \sigma(a)) z^{*} \\
& -\psi(a) \oplus \eta(a) \oplus \sigma(a) \| \\
& <2 \epsilon / 3+\epsilon / 3=\epsilon .
\end{aligned}
$$

Proposition 2.3 ([D3]). Let $A$ be a unital separable $R F D C^{*}$-algebra. Then $A$ is nuclearly embeddable if and only if for any unital faithful representation $\sigma: A \rightarrow \mathcal{L}(\mathcal{H})$ with $\sigma(A) \cap \mathcal{K}(\mathcal{H})=\{0\}$, there exists a sequence of unital representations $\rho_{n}: A \rightarrow \mathcal{L}(\mathcal{H})$ whose images are contained in finite dimensional $C^{*}$-subalgebras of $\mathcal{L}(\mathcal{H})$ and such that for all $a \in A, \lim _{n \rightarrow \infty}\left\|\sigma(a)-\rho_{n}(a)\right\|=0$ 
Proof. This was proved in [D3]. A different proof is given the Appendix.

Definition 2.4. Let $A$ be a unital RFD $\mathrm{C}^{*}$-algebra. Let $\mathcal{F} \subset A$ be a finite subset and let $\epsilon>0$. A unital representation $\pi: A \rightarrow M_{k}$ is called $(\mathcal{F}, \epsilon)$-admissible if there is a unital faithful representation $\sigma: A \rightarrow \mathcal{L}(\mathcal{H})$ with $\sigma(A) \cap \mathcal{K}(\mathcal{H})=\{0\}$, $\left(\mathcal{H}=\mathbb{C}^{k} \oplus \mathbb{C}^{k} \oplus \cdots\right)$ such that if $\pi_{\infty}=\pi \oplus \pi \oplus \cdots$, then

$$
\left\|\sigma(a)-\pi_{\infty}(a)\right\|<\epsilon \quad a \in \mathcal{F} .
$$

Note that if $\pi$ is $(\mathcal{F}, \epsilon)$-admissible, then so is $\pi \oplus \gamma$ for any unital finite dimensional representation $\gamma$. Moreover $\|\pi(a)\| \geq\|a\|-\epsilon$ for $a \in \mathcal{F}$. If $A$ is separable nuclearly embeddable and RFD, then Proposition 2.3 guaranties the existence of $(\mathcal{F}, \epsilon)$-admissible representations for any finite set $\mathcal{F} \subset A$ and any $\epsilon>0$. The following proposition is crucial for our embedding result. If $n$ is a positive integer and $\pi$ is a representation, then $n \pi$ will denote the representation $\pi \oplus \cdots \oplus \pi$ (n-times).

Proposition 2.5. Let $A$ be a separable unital nuclearly embeddable $R F D C^{*}$ algebra. Let $\mathcal{F} \subset A$ be a finite subset and let $\epsilon>0$. Then for any $(\mathcal{F}, \epsilon)$ admissible representation $\pi: A \rightarrow M_{k}$ and any two homotopic unital representations $\varphi, \psi: A \rightarrow M_{m}$, there exist a positive integer $N$ and a unitary $u \in M_{m+N k}$ such that

$$
\left\|u(\varphi(a) \oplus N \pi(a)) u^{*}-\psi(a) \oplus N \pi(a)\right\|<3 \epsilon, \quad a \in \mathcal{F} .
$$

Proof. By definition, $\pi$ satisfies (8) for some unital faithful representation $\sigma$ : $A \rightarrow \mathcal{L}(\mathcal{H})$ with $\sigma(A) \cap \mathcal{K}(\mathcal{H})=\{0\}$. By applying Proposition 2.2 to $\varphi$ and $\psi$ we find a unitary $v \in \mathbb{C} 1_{\mathbb{C}^{m} \oplus \mathcal{H}}+\mathcal{K}\left(\mathbb{C}^{m} \oplus \mathcal{H}\right)$ such that

$$
\left\|v(\varphi(a) \oplus \sigma(a)) v^{*}-\psi(a) \oplus \sigma(a)\right\|<\epsilon, \quad a \in \mathcal{F} .
$$

From (8) and (9) we then obtain

$$
\left\|v\left(\varphi(a) \oplus \pi_{\infty}(a)\right) v^{*}-\psi(a) \oplus \pi_{\infty}(a)\right\|<3 \epsilon, \quad a \in \mathcal{F} .
$$

Let $\mathcal{H}_{n}=\mathbb{C}^{m} \oplus \mathbb{C}^{k} \oplus \cdots \oplus \mathbb{C}^{k} \subset \mathbb{C}^{m} \oplus \mathcal{H}\left(n\right.$ copies of $\left.\mathbb{C}^{k}\right)$ and let $e_{n}$ denote the orthogonal projection of $\mathbb{C}^{m} \oplus \mathcal{H}$ onto $\mathcal{H}_{n}$. After a small perturbation of $v$ we may assume that $v \in \mathbb{C} 1_{\mathbb{C}^{m} \oplus \mathcal{H}}+\mathcal{K}\left(\mathcal{H}_{N}\right)$ for some large $N$. It is then clear that $e_{N}$ commutes with $v$ and with the images of $\varphi \oplus \pi_{\infty}$ and $\psi \oplus \pi_{\infty}$. Then $e_{N}\left(\varphi \oplus \pi_{\infty}\right) e_{N}=\varphi \oplus N \pi, e_{N}\left(\psi \oplus \pi_{\infty}\right) e_{N}=\psi \oplus N \pi$ and $u=e_{N} v e_{N}$ is a unitary in $\mathcal{L}\left(\mathcal{H}_{N}\right) \cong M_{m+N k}$. We finish the proof by compressing by $e_{N}$ in (10).

For a $\mathrm{C}^{*}$-algebra $A$ we denote by $C A$ the cone of $A\left(C A=C_{0}[0,1) \otimes A\right)$ and by $S A$ the suspension of $A\left(S A=C_{0}(0,1) \otimes A\right)$. Let $\widetilde{A}$ denote the $\mathrm{C}^{*}$-algebra obtained by adding a unit to $A$.

Theorem 2.6. Let $A$ be a separable nuclearly embeddable RFD $C^{*}$-algebra. Then $C A$ and $S A$ are embeddable in the $C A R$ algebra $B$. 
Proof. We have that $S A \subset C A \subset \widetilde{C A}$ so that it suffices to show that $D=$ $\widetilde{C A}$ embeds unitally in $B$. A key property of $D$ is that any two unital *homomorphisms $D \rightarrow M_{k}$ are homotopic. Let $\left(\mathcal{F}_{n}\right)$ be a sequence of increasing finite subsets of $D$ whose union is dense in $D$ and let $\epsilon_{n}=2^{-n}$. We will construct inductively a sequence $(r(n))$ of powers of two and a sequence $\left(\gamma_{n}\right)$ of representations $\gamma_{n}: D \rightarrow M_{k(n)}$, where $k(1)=r(1), k(n)=k(n-1) r(n)$ for $n \geq 2$, such that

(i) $\gamma_{n}$ is $\left(\mathcal{F}_{n}, \epsilon_{n}\right)$-admissible (in particular $\left\|\gamma_{n}(a)\right\| \geq\|a\|-\epsilon_{n}$ for $a \in \mathcal{F}_{n}$ ).

(ii) $\left\|\gamma_{n}(a)-r(n) \gamma_{n-1}(a)\right\|<3 \epsilon_{n-1}$, for $a \in \mathcal{F}_{n-1}$.

Let $\gamma_{1}: D \rightarrow M_{k(1)}$ be an $\left(\mathcal{F}_{1}, \epsilon_{1}\right)$-admissible representation. Such representations exist by Proposition 2.3 since $D$ is nuclearly embeddable and RFD. By adding one-dimensional representations to $\gamma_{1}$ we may arrange that $k(1)$ is a power of two. Suppose now that $\gamma_{1}, \ldots, \gamma_{n}$ and $r(1), \ldots, r(n)$ were constructed. Let $\pi: D \rightarrow M_{k}$ be an $\left(\mathcal{F}_{n+1}, \epsilon_{n+1}\right)$-admissible representation. By adding onedimensional representations to $\pi$ we can assume that $k=s k(n)$ for some integer $s$. Then $\pi(1)=s \gamma_{n}(1)$ hence $\pi$ and $s \gamma_{n}$ are homotopic. Since $\gamma_{n}$ is $\left(\mathcal{F}_{n}, \epsilon_{n}\right)$ admissible, by Proposition 2.5 there is $N$ and a unitary $u \in M_{k+N k(n)}$ such that $\left\|u\left(\pi(a) \oplus N \gamma_{n}(a)\right) u^{*}-s \gamma_{n}(a) \oplus N \gamma_{n}(a)\right\|<3 \epsilon_{n}$ for $a \in \mathcal{F}_{n}$. By increasing $N$ we may arrange that $N+s$ is a power of two. We conclude the construction by defining $r(n+1)=N+s$ and $\gamma_{n+1}(a)=u\left(\pi(a) \oplus N \gamma_{n}(a)\right) u^{*}$. Let $\iota_{n}: M_{k(n)} \hookrightarrow B$ be the canonical inclusion. Having the sequence $\gamma_{n}$ available, we construct a unital embedding $\gamma: D \rightarrow \lim M_{k(n)} \cong B$ by defining $\gamma(a), a \in \cup_{n} \mathcal{F}_{n}$, to be the limit of the Cauchy sequence $\left(\iota_{n} \gamma_{n}(a)\right)$ and then extend to $D$ by continuity. Note that $\|\gamma(a)\|=\|a\|$ since $\left\|\gamma_{n}(a)\right\| \geq\|a\|-\epsilon_{n}$ for $a \in \mathcal{F}_{n}$.

\section{Subquotients of the CAR algebra}

Lemma 3.1. Let $A$ be a separable unital nuclearly embeddable $C^{*}$-algebra. Then there exists a semisplit essential extension $0 \rightarrow I \rightarrow \widetilde{C F} \rightarrow A \rightarrow 0$ with $F$ a unital separable nuclearly embeddable RFD $C^{*}$-algebra.

Proof. Since $\widetilde{C A}$ is homotopic to $\mathbb{C}$, any unital representation $\sigma: D \rightarrow \mathcal{L}(\mathcal{H})$ of $D=\widetilde{C A}$ is homotopic to a representation with image contained in $\mathbb{C} 1_{\mathcal{H}}$. By [Vo3, Proposition 3] (its proof rather than its statement), there is a unital *-monomorphism $j: D \rightarrow \prod_{n=1}^{\infty} M_{k(n)} / \sum_{n=1}^{\infty} M_{k(n)}$ which admits a unital completely positive lifting $\eta: D \rightarrow \prod M_{k(n)}$. Consider the diagram

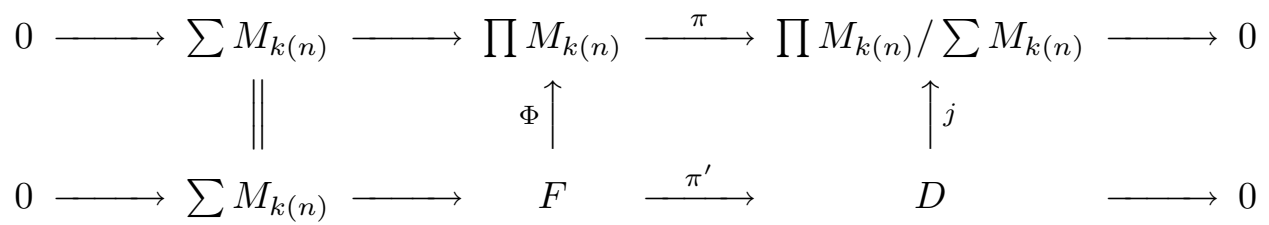

where the extension at the bottom is the pull-back of the extension at the top. We are going to show that the unital $*$-monomorphism $\Phi$ is nuclear, hence $F$ is a nuclearly embeddable $\mathrm{C}^{*}$-algebra. Since $D$ is nuclearly embeddable, the map 
$\eta$ is nuclear by [D1, Proposition 3.3]. Note that $\eta$ induces a unital completely positive map $\eta^{\prime}: D \rightarrow F$ such that $\pi^{\prime} \eta^{\prime}=i d_{D}$ and $\Phi \eta^{\prime}=\eta$. Let $e_{n}$ be the unit of $M_{k(1)} \oplus \cdots \oplus M_{k(n)}$. Then $\left(e_{n}\right)$ is an approximate unit of projections of $\sum M_{k(n)}$ which is central in $\prod M_{k(n)}$ hence it is central in $F$. For any $z \in F$, $z=e_{n} z e_{n}+\left(1-e_{n}\right) z\left(1-e_{n}\right)$ and

$$
\begin{aligned}
\lim _{n \rightarrow \infty}\left\|z-e_{n} z e_{n}-\left(1-e_{n}\right) \eta^{\prime}\left(\pi^{\prime}(z)\right)\left(1-e_{n}\right)\right\| & = \\
\lim _{n \rightarrow \infty}\left\|\left(1-e_{n}\right)\left(z-\eta^{\prime}\left(\pi^{\prime}(z)\right)\right)\left(1-e_{n}\right)\right\| & =0
\end{aligned}
$$

as $z-\eta^{\prime}\left(\pi^{\prime}(z)\right) \in \sum M_{k(n)}$. Since $\Phi \eta^{\prime}=\eta$, we obtain

$$
\lim _{n \rightarrow \infty}\left\|\Phi(z)-e_{n} \Phi(z) e_{n}-\left(1-e_{n}\right) \eta\left(\pi^{\prime}(z)\right)\left(1-e_{n}\right)\right\|=0 .
$$

This proves that $\Phi$ is nuclear since the maps $\eta$ and $e_{n} \Phi(-) e_{n}$ are nuclear. Note that $F$ is RFD as it embeds in $\prod M_{k(n)}$.

To finish the proof we need the following observation. Suppose that $G$ is a unital $C^{*}$-algebra. Then $\widetilde{C G} \cong\left\{f \in C([0,1], G): f(1) \in \mathbb{C} 1_{G}\right\}$. Let $\lambda$ be a state of $G$. The surjection $\pi_{G}: \widetilde{C G} \rightarrow G, \pi_{G}(f)=f(0)$ admits a unital completely positive right inverse given by $\eta_{G}(a)(t)=t \lambda(a) 1_{G}+(1-t) a,(a \in G, t \in[0,1])$. It follows that $\eta_{F} \circ \eta^{\prime} \circ \eta_{A}$ is a unital completely positive right inverse for the composition

$$
\widetilde{C F} \stackrel{\pi_{F}}{\longrightarrow} F \stackrel{\pi^{\prime}}{\longrightarrow} \widetilde{C A} \stackrel{\pi_{A}}{\longrightarrow} A
$$

Moreover it is clear that $\widetilde{C F}$ is nuclearly embeddable and RFD since $F$ is so. Finally we set $I=\operatorname{ker}\left(\pi_{A} \circ \pi^{\prime} \circ \pi_{F}\right)$ and notice that $I$ is an essential ideal of $\widetilde{C F}$ as it contains $S F$.

\section{Proof of Theorem 1.1}

(i) $\Rightarrow$ (ii) Let $0 \rightarrow I \rightarrow \widetilde{C F} \stackrel{\nu}{\longrightarrow} A \rightarrow 0$ be the extension given Lemma 3.1 and let $\eta$ be a unital completely positive right inverse of $\nu$. By Theorem 2.6 $\widetilde{C F}$ embeds unitally in $B$. Let $J=\overline{I B I}$ be the hereditary $\mathrm{C}^{*}$-subalgebra of $B$ generated by $I$. Then $J$ is a two-sided closed ideal of the $\mathrm{C}^{*}$-algebra $E=\widehat{C F}+J$ and it is easy to check that we still have a semisplit essential unital extension $0 \rightarrow J \rightarrow E \stackrel{\pi}{\longrightarrow} A \rightarrow 0$. Indeed, the composition of $\widetilde{C F} \hookrightarrow E$ with $\eta$ defines a unital completely positive right inverse of $\pi$. To check that $J$ is essential in $E$, let $x \in \widetilde{C F}, y \in J$ be such that $(x+y) J=0$. Let $\left(h_{n}\right)$ be an approximate unit of $I$. Then $h_{n}(x+y) h_{n}=0$ hence $y=-\lim _{n \rightarrow \infty} h_{n} x h_{n} \in I$. Now $(x+y) I=0$ implies $x+y=0$ since $I$ is essential in $\widetilde{C F}$. Since $J$ is a hereditary $\mathrm{C}^{*}$-subalgebra of $B$ and $B$ is simple, $J$ is stably isomorphic to $B$ by Brown's theorem [Br]. The rest of the proof is taken from [W3]. We include it for the sake of completeness. (ii) $\Rightarrow$ (i) Let $\eta: A \rightarrow E$ be a unital completely positive right inverse of $\pi: E \rightarrow A$. Since $\pi \eta=i d_{A}, \eta$ is a unital complete isometry hence it is a complete order embedding. This means that its inverse $\eta^{-1}: \eta(A) \rightarrow A$ is unital and completely positive. Let $\sigma: A \rightarrow \mathcal{L}(\mathcal{H})$ be a 
unital faithful representation. By Arveson's extension theorem $\sigma \eta^{-1}$ extends to a unital completely positive map $\theta: B \rightarrow \mathcal{L}(\mathcal{H})$. Then $\sigma=\theta \eta$ is nuclear since it factorises through the nuclear algebra $B$ (ii) $\Rightarrow$ (iii) is obvious. (iii) $\Rightarrow$ (ii) If $E$ embeds in $B$, then so does $\widetilde{C E}$ since $C_{0}[0,1) \subset B$ and $B \otimes B \cong B$. After replacing the map $E \rightarrow A$ by the composition $\widetilde{C E} \rightarrow E \rightarrow A$ whose kernel is an essential ideal of $\widetilde{C E}$, we may assume that the given extension is essential. Arguing as in the last part of (i) $\Rightarrow$ (ii), we may arrange that $J$ is an essential AF-ideal. Since $B$ has property $(C)$ of Archbold and Batty and $E \subset B, E$ has property $(C)[\mathrm{AB}]$. One concludes the proof by applying the lifting results of Effros and Haagerup. Indeed, by [EK, Proposition 5.3 (3) and Theorem 3.4] any extension $0 \rightarrow J \rightarrow E \rightarrow A \rightarrow 0$ is semisplit if $E$ has property (C) and $J$ is an AF-ideal.

\section{Applications}

Following Kirchberg, let us review the main applications of Theorem 1.1.

1. Any separable unital nuclearly embeddable $C^{*}$-algebra embeds as a unital $C^{*}$ subalgebra of the Cuntz algebra $\mathcal{O}_{2}$. We use the implication (i) $\Rightarrow$ (ii) of Theorem 1.1 so that we do not rely on [EK]. Let $0 \rightarrow J \rightarrow E \rightarrow A \rightarrow 0$ be a semisplit essential extension given by Theorem 1.1. Since $B$ embeds unitally in $\mathcal{O}_{2}$ [Cu1, 1.5], after replacing $J$ by the hereditary $\mathrm{C}^{*}$-subalgebra $J^{\prime}$ of $\mathcal{O}_{2}$ generated by $J$ and replacing $E$ by $E^{\prime}=E+J^{\prime}$ we obtain a semisplit essential extension $0 \rightarrow J^{\prime} \rightarrow E^{\prime} \rightarrow A \rightarrow 0$ where $J^{\prime}$ is stably isomorphic to $\mathcal{O}_{2}$ by $[\mathrm{Br}]$. In particular $J^{\prime}$ is non-unital and hence stable by [Zh, Theorem 1.2] so that $J^{\prime} \cong \mathcal{O}_{2} \otimes \mathcal{K}(\mathcal{H})$. By the Weyl-von Neumann-Voiculescu type theorem of Kirchberg [Ki4], the extension $0 \rightarrow J^{\prime} \rightarrow E^{\prime} \rightarrow A \rightarrow 0$ is unitally absorbing. Since $\operatorname{Ext}^{-1}\left(A, \mathcal{O}_{2}\right)=0$, (as $i d_{\mathrm{O}_{2}} \oplus i d_{\mathrm{O}_{2}}$ is homotopic to $i d_{\mathcal{O}_{2}}$ by [Cu2] and $\operatorname{Ext}^{-1}(A,-)$ is homotopy invariant by [Ka]) we conclude that the extension $0 \rightarrow J^{\prime} \rightarrow E^{\prime} \rightarrow A \rightarrow 0$ splits. Therefore there is a unital $*$-monomorphism $\gamma: A \rightarrow E^{\prime} \subset \mathcal{O}_{2}$.

2. Any quotient of a unital separable nuclearly embeddable $C^{*}$-algebra $A$ is nuclearly embeddable. This is an obvious consequence of the equivalence between (i) and (iii), whose proof relies essentially on [EK].

\section{Appendix}

Here we prove a generalization of Proposition 2.3 which clarifies the role of nuclear embeddability in our context. Let $A$ be a $\mathrm{C}^{*}$-algebra, let $\mathcal{F} \subset A$ be a finite subset and let $\epsilon>0$. If $\varphi: A \rightarrow \mathcal{L}\left(\mathcal{H}_{\varphi}\right)$ and $\psi: A \rightarrow \mathcal{L}\left(\mathcal{H}_{\psi}\right)$ are two maps, we write $\varphi \underset{\mathcal{F}, \epsilon}{\prec} \psi$ if there is an isometry $v: \mathcal{H}_{\varphi} \rightarrow \mathcal{H}_{\psi}$ such that $\left\|\varphi(a)-v^{*} \psi(a) v\right\|<\epsilon$ for all $a \in \mathcal{F}$. If $v$ can be chosen to be a unitary, then we

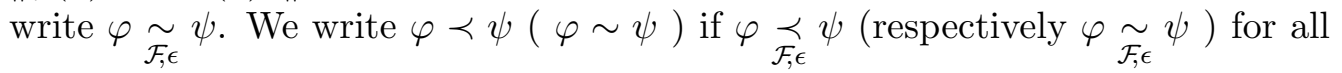

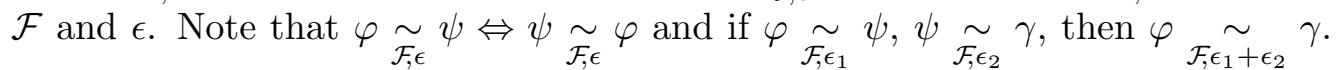
We let $\varphi_{\infty}$ denote the infinite direct $\operatorname{sum} \varphi \oplus \varphi \oplus \cdots$. 
Lemma 5.1. Let $A$ be a unital $C^{*}$-algebra, let $\mathcal{F} \subset A$ be a finite subset and let $\epsilon>0$. There exist $\mathcal{G} \subset A$ a finite subset and $\delta>0$ such that if $\varphi: A \rightarrow \mathcal{L}\left(\mathcal{H}_{\varphi}\right)$, $\psi: A \rightarrow \mathcal{L}\left(\mathcal{H}_{\psi}\right)$ are selfadjoint contractions with $\left\|\varphi\left(a^{*} a\right)-\varphi\left(a^{*}\right) \varphi(a)\right\|<\delta$, $\left\|\psi\left(a^{*} a\right)-\psi\left(a^{*}\right) \psi(a)\right\|<\delta, a \in \mathcal{G}$, then we have the following. (i) If $\varphi_{\infty} \underset{\mathcal{G}, \delta}{\prec} \psi$, then $\varphi \oplus \psi \underset{\mathcal{F}, \epsilon}{\widetilde{\mathcal{F}}} \psi$. (ii) If $\varphi_{\infty} \underset{\mathcal{G}, \delta}{\prec} \psi$ and if $\psi_{\infty} \underset{\mathcal{G}, \delta}{\prec} \varphi$, then $\varphi_{\underset{\mathcal{F}, \epsilon}{\widetilde{f}}}^{\sim}$.

Proof. This goes along the lines of the proof of Voiculescu's theorem [Vo1], [Ar]. It suffices to prove only part (i), since (i) $\Rightarrow$ (ii). Let $\mathcal{G}=\left\{a b: a, b \in \mathcal{F} \cup \mathcal{F}^{*}\right\} \cup$ $\mathcal{F} \cup \mathcal{F}^{*}$ and let $\delta>0$ be small enough so that $2 \delta+8 \delta^{1 / 2}(2 M+3)^{1 / 2}<\epsilon$, where $M=\max \{\|a\|: a \in \mathcal{F}\}$. Define $\phi=\varphi_{\infty}$. By assumption there is an isometry $v: \mathcal{H}_{\phi} \rightarrow \mathcal{H}_{\psi}$ such that

$$
\left\|\phi(x)-v^{*} \psi(x) v\right\|<\delta, \quad x \in \mathcal{G} .
$$

From (11) and the identity

$$
\begin{aligned}
(v \phi(a)-\psi(a) v)^{*}(v \phi(a)-\psi(a) v) & =\phi\left(a^{*}\right)\left(\phi(a)-v^{*} \psi(a) v\right)+\left(\phi\left(a^{*}\right)\right. \\
& \left.-v^{*} \psi\left(a^{*}\right) v\right) \phi(a)+v^{*}\left(\psi\left(a^{*}\right) \psi(a)\right. \\
& \left.-\psi\left(a^{*} a\right)\right) v+\left(v^{*} \psi\left(a^{*} a\right) v\right. \\
& \left.-\phi\left(a^{*} a\right)\right)+\left(\phi\left(a^{*} a\right)-\phi\left(a^{*}\right) \phi(a)\right)
\end{aligned}
$$

we obtain

$$
\|v \phi(a)-\psi(a) v\|<\delta^{1 / 2}(2 M+3)^{1 / 2}, \quad a \in \mathcal{F} \cup \mathcal{F}^{*} .
$$

If $p=v v^{*}$, then since $\phi$ is selfadjoint

$$
[\psi(a), p]=(\psi(a) v-v \phi(a)) v^{*}+v\left(v \phi\left(a^{*}\right)-\psi\left(a^{*}\right) v\right)^{*} .
$$

From (12) and (13) we obtain $\|\psi(a) p-p \psi(a)\|<2 \delta^{1 / 2}(2 M+3)^{1 / 2}$ for all $a \in \mathcal{F}$, hence

(14) $\|\psi(a)-p \psi(a) p-(1-p) \psi(a)(1-p)\|<\delta_{1}=4 \delta^{1 / 2}(2 M+3)^{1 / 2}, \quad a \in \mathcal{F}$.

Regarding $v$ as a unitary from $\mathcal{H}_{\phi}$ to $p \mathcal{H}_{\psi}$, we obtain from (11)

$$
\phi \underset{\mathcal{G}, \delta}{\sim} p \psi p .
$$

Combining (14) with (15) and setting $\lambda(a)=(1-p) \psi(a)(1-p)$ we have $\phi \oplus \lambda \underset{\mathcal{G}, \delta}{\widetilde{1}, p} p p \oplus(1-p) \psi(1-p) \underset{\mathcal{F}, \delta_{1}}{\sim} \psi$, hence

$$
\varphi_{\infty} \oplus \lambda=\phi \oplus \lambda \underset{\mathcal{F}, \epsilon / 2}{\sim} \psi
$$

since $\mathcal{F} \subset \mathcal{G}$ and $\delta+\delta_{1}=\delta+4 \delta^{1 / 2}(2 M+3)^{1 / 2}<\epsilon / 2$ by our choice of $\delta$. Therefore

$$
\psi \underset{\mathcal{F}, \epsilon / 2}{\sim} \varphi_{\infty} \oplus \lambda \sim \varphi \oplus \varphi_{\infty} \oplus \lambda \underset{\mathcal{F}, \epsilon / 2}{\sim} \varphi \oplus \psi
$$

hence $\psi \underset{\mathcal{F}, \epsilon}{\sim} \varphi \oplus \psi$. 


\section{Proof of Proposition 2.3}

The result is a consequence of the following.

Proposition 6.1. Let $A$ be a unital separable nuclearly embeddable $C^{*}$-algebra and let $\left(\chi_{n}\right)$ be a sequence of unital representations separating the elements of $A$. Then for any $\mathcal{F} \subset A$ a finite subset and any $\epsilon>0$ there is a representation $\pi$ of $A$ of the form $\pi=\chi_{i_{1}} \oplus \chi_{i_{2}} \oplus \cdots \oplus \chi_{i_{m}}$ such that if $\sigma: A \rightarrow \mathcal{L}(\mathcal{H})$ is any unital faithful representation with $\sigma(A) \cap \mathcal{K}(\mathcal{H})=\{0\}$, then $\sigma \underset{\mathcal{F}, \epsilon}{\widetilde{\pi_{\infty}}} \pi_{\text {. }}$

Proof. By Voiculescu's theorem [Vo1] it suffices to prove the statement for a fixed representation $\sigma$. With $\mathcal{F}$ and $\epsilon$ as above let $\mathcal{G} \subset A$ and $\delta>0$ be given by Lemma 5.1. In the first part of the proof we find $\pi$ of the desired form such that $\sigma \underset{\mathcal{G}, \delta}{\prec} \pi_{\infty}$. Since $A$ is nuclearly embeddable, the representation $\sigma$ is nuclear. Thus we find unital completely positive maps $\alpha: A \rightarrow \mathcal{L}\left(\mathbb{C}^{k}\right)$ and $\beta: \mathcal{L}\left(\mathbb{C}^{k}\right) \rightarrow \mathcal{L}(\mathcal{H})$ such that

$$
\|\sigma(a)-\beta \alpha(a)\|<\delta / 2, \quad a \in \mathcal{G} .
$$

We may assume that each representation in the sequence $\left(\chi_{n}\right)$ repeats itself infinitely many times. Thus $\chi=\chi_{1} \oplus \chi_{2} \oplus \ldots$ is a unital faithful representation of $A$ of infinite multiplicity. If $\pi_{\alpha}$ is the Stinespring dilation of $\alpha$, then we have $\alpha \prec \pi_{\alpha} \prec \pi_{\alpha} \oplus \chi$. Therefore $\alpha \prec \chi$ since $\pi_{\alpha} \oplus \chi \sim \chi$ by Voiculescu's theorem. By a standard perturbation argument we obtain $\alpha \underset{\mathcal{G}, \delta / 2}{\prec} \chi_{1} \oplus \cdots \oplus \chi_{n}$ for some large enough $n$. Thus if we set $\pi=\chi_{1} \oplus \cdots \oplus \chi_{n}$, then there is an isometry $w: \mathbb{C}^{k} \rightarrow \mathcal{H}_{\pi}$ with

$$
\left\|\alpha(a)-w^{*} \pi(a) w\right\|<\delta / 2, \quad a \in \mathcal{G} .
$$

By Stinespring's theorem, the unital completely positive map $x \mapsto \beta\left(w^{*} x w\right)$ can be dilated to a unital representation $\rho: \mathcal{L}\left(\mathcal{H}_{\pi}\right) \rightarrow \mathcal{L}\left(\mathcal{H}_{\rho}\right)$. Thus we find an isometry $v: \mathcal{H} \rightarrow \mathcal{H}_{\rho}$ with

$$
\beta\left(w^{*} x w\right)=v^{*} \rho(x) v, \quad x \in \mathcal{L}\left(\mathcal{H}_{\pi}\right) .
$$

From (16), (17) and (18) we obtain

$$
\begin{array}{r}
\left\|\sigma(a)-v^{*} \rho(\pi(a)) v\right\|=\left\|\sigma(a)-\beta\left(w^{*} \pi(a) w\right)\right\| \\
\leq\|\sigma(a)-\beta \alpha(a)\|+\left\|\beta \alpha(a)-\beta\left(w^{*} \pi(a) w\right)\right\|<\delta / 2+\delta / 2=\delta, \quad a \in \mathcal{G} .
\end{array}
$$

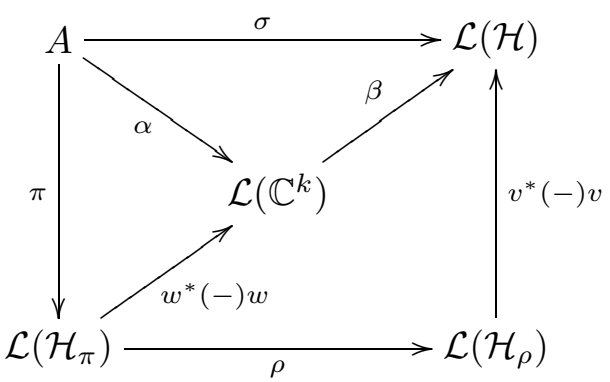


This gives $\sigma \underset{\mathcal{G}, \delta}{\prec} \rho \pi$. It is clear from the representation theory of $\mathcal{L}\left(\mathcal{H}_{\pi}\right)$ that $\rho \pi \sim \pi_{\infty}$. Therefore $\sigma \underset{\mathcal{G}, \delta}{\prec} \pi_{\infty}$ hence $\sigma_{\infty} \underset{\mathcal{G}, \delta}{\prec} \pi_{\infty}$ as $\left(\pi_{\infty}\right)_{\infty} \sim \pi_{\infty}$. By Voiculescu's theorem, we have $\sigma \oplus \pi_{\infty} \sim \sigma$ hence $\left(\pi_{\infty}\right)_{\infty} \prec \sigma$. By Lemma 5.1(ii) it follows that $\sigma \underset{\mathcal{F}, \epsilon}{\sim} \pi_{\infty}$.

\section{References}

[AB] R. J. Archbold and C. J. K. Batty, $C^{*}$-tensor norms and slice maps, J. London Math. Soc. (2) 22 (1980), no. 1, 127-138.

[Ar] W. B. Arveson Notes on extensions of $C^{*}$-algebras, Duke Math. J. 44 (1977), 329-355.

[Bla] B. Blackadar, Nonnuclear subalgebras of $C^{*}$-algebras, J. Operator Theory, 14 (1985), 347-350.

[Br] L.G. Brown, Stable isomorphism of hereditary subalgebras of $C^{*}$-algebras, Pacific J. Math. 71 (1977), no. 2, 335-348.

[Cu1] J. Cuntz, Simple $C^{*}$-algebras generated by isometries, Comm. Math. Phys. 57 (1977), 173-185.

[Cu2] J. Cuntz, K-theory for certain $C^{*}$-algebras, Ann. of Math. 113 (1981), 181-197.

[D1] M. Dadarlat, Quasidiagonal morphisms and homotopy J. Funct. Anal. 151 (1997), no. $1,213-233$.

[D2] M. Dadarlat, Residually finite-dimensional $C^{*}$-algebras, Operator algebras and operator theory (Shanghai, 1997), 45-50, Contemp. Math., 228, Amer. Math. Soc., Providence, RI, 1998.

[D3] M. Dadarlat On the approximation of quasidiagonal $C^{*}$-algebras, J. Funct. Anal. 167 (1999), no. 1 69-78.

[D4] M. Dadarlat, Embeddings of nuclearly embeddable $C^{*}$-algebras, Preprint March 2000.

[DE] M. Dadarlat and S. Eilers, Asymptotic unitary equivalence in KK-theory, to appear in K-theory.

[EK] E. G. Effros and U. Haagerup, Lifting problems and local reflexivity for $C^{*}$-algebras Duke Math. J. 52 (1985), no. 1, 103-128.

[Ka] G.G. Kasparov, The operator $K$-functor and extensions of $C^{*}$-algebras, Izv. Akad. Nauk SSSR Ser. Mat. 44 (1980), no. 3, 571-636, 719.

[Ki1] E. Kirchberg, On restricted perturbations in inverse images and a description of normalizer algebras in $C^{*}$-algebras J. Funct. Anal. 129 (1995), no. 1, 1-34.

[Ki2] E. Kirchberg, On subalgebras of the CAR-algebra J. Funct. Anal. 129 (1995), no. 1, 35-63.

[Ki3] E. Kirchberg, Commutants of unitaries in UHF algebras and functorial properties of exactness. J. Reine Angew. Math. 452 (1994), 39-77.

[Ki4] E. Kirchberg, The classification of purely infinite $C^{*}$-algebras using Kasparov's theory, preprint, third draft, 1994.

[KPh] E. Kirchberg and N. C. Phillips, Embedding of exact $C^{*}$-algebras in the Cuntz algebra $\mathcal{O}_{2}$. J. Reine Angew. Math. 525 (2000), 17-53.

[L] H. Lin, Residually finite dimensional and AF-embeddable $C^{*}$-algebras. Proc. Amer. Math. Soc. 129 (2001), no. 6, 1689-1696 .

[RS] J. Rosenberg and C. Schochet, The Künneth theorem and the universal coefficient theorem for Kasparov's generalized K-functor, Duke Math. J. 55 (1987), 431-474.

[Sk] G. Skandalis, Une notion de nucléarité en K-théorie (d'après J. Cuntz), K-Theory 1 (1988), 549-573.

[Vo1] D. Voiculescu, A non-commutative Weyl-von Neumann theorem, Rev. Roumaine Math. Pures Appl. 21 (1976), no. 1, 97-113.

[Vo2] D. Voiculescu, A note on quasidiagonal operators Topics in operator theory, 265-274, Oper. Theory: Adv. Appl., 32, Birkhäuser, Basel-Boston, MA, 1988. 
[Vo3] D. Voiculescu, A note on quasi-diagonal $C^{*}$-algebras and homotopy. Duke Math. J. 62 (1991), no. 2, 267-271.

[W1] S. Wassermann, Subquotients of UHF $C^{*}$-algebras. Math. Proc. Cambridge Philos. Soc. 115 (1994), no. 3, 489-500.

[W2] S. Wassermann, Tensor products of free-group $C^{*}$-algebras Bull. London Math. Soc. 22 (1990), no. 4, 375-380.

[W3] S. Wassermann, Exact $C^{*}$-algebras and related topics Lecture Notes Series, 19. Seoul National University, Research Institute of Mathematics, Global Analysis Research Center, Seoul, 1994. viii+92 pp.

[Zh] S. Zhang, Certain $C^{*}$-algebras with real rank zero and their corona and multiplier algebras. I Pacific J. Math. 155 (1992), no. 1, 169-197.

Department of Mathematics, Purdue University, West Lafayette IN 47907.

E-mail address: mdd@math.purdue.edu 